\title{
Detection of Maternal Alcohol Use Problems in the Pediatric Emergency Department
}

\author{
Heather A. Flynn, Sarah A. Cain, Heather A. O’Mahen, and Matthew M. Davis
}

\begin{abstract}
Background: Maternal alcohol use problems may impact the health and well-being of children, but often remain unrecognized. Mothers of young children seldom seek outpatient care for themselves; thus, pediatric settings may present an opportunity for the detection of maternal alcohol use problems. This study examines the feasibility of screening for and prevalence of alcohol use problems in mothers of young children in the context of seeking pediatric emergency care. We also examined the relationship of maternal alcohol use problems with use of pediatric emergency care.

Methods: A total of 361 English-speaking mothers of children aged 7 and younger completed screening measures during their child's emergency care visit. TWEAK was used to screen for alcohol use problems. The screening survey also included information on children's health status and health care use, demographics, and the Center for Epidemiological Studies Depression Scale.

Results: Of the women approached, $90 \%$ agreed to complete the screening measure. On the basis of cutoff score of 2 or more, $7 \%$ of women had elevated TWEAK scores. Those women with a TWEAK score $>2$ reported greater use of the pediatric emergency department (PED) than women scoring below the cutoff. On the basis of multivariate analyses, significant predictors of recent PED use included the presence of child chronic illness, younger maternal age, and TWEAK score.

Conclusions: Screening for alcohol use problems among mothers of young children using the TWEAK appears to be feasible in a busy PED setting. The PED setting is promising for identifying risk drinking among women who may be less likely to be otherwise detected and for whom alcohol use may be impacting child outcomes.
\end{abstract}

Key Words: Maternal Drinking, TWEAK, Emergency Department.

$\mathrm{T}$ HIS STUDY AIMED to demonstrate the feasibility of screening for alcohol use problems in mothers of young children in the context of seeking pediatric emergency care, the prevalence of maternal alcohol use in this setting, and the relationship of maternal alcohol use problems with use of pediatric emergency care. More severe alcohol use problems, such as alcohol dependence, in mothers have been linked to a number of negative child outcomes, including physical, academic, social, and emotional problems (Conners et al., 2004). Women of child-bearing age have higher prevalence rates than women of other ages for alcohol abuse or dependence nearly $10 \%$ for women aged 18 to 29 and $4 \%$ for women aged 30 to 44 (Larkby and Day, 1999; Richter and Richter,

From the Department of Psychiatry, University of Michigan Medical School, Ann Arbor, Michigan (HAF, SAC, HAO); the Departments of Pediatrics and Internal Medicine, University of Michigan Medical School, Ann Arbor, Michigan (MMD); and the Gerald R. Ford School of Public Policy, University of Michigan, Ann Arbor, Michigan.

Received for publication July 22, 2005; accepted February 23, 2006.

This work was supported by the Rachel Upjohn Clinical Scholars Award.

Reprint requests: Heather A. Flynn, PhD, Department of Psychiatry, University of Michigan, 2101 Commonwealth Road, Suite B, Ann Arbor, MI 48105; E-mail:hflynn@umich.edu

Copyright (C) 2006 by the Research Society on Alcoholism.

DOI: 10.1111/j.1530-0277.2006.00132.x
2001). Despite the vast literature on the impact of parental alcohol dependence on an array of negative child outcomes (for reviews see Sher, 1991; Harter, 2000), there is less known about the effects of less severe maternal alcohol use problems on child health indicators. The importance of parental psychosocial factors on child health care use has received growing recognition, and pediatric screening for alcohol use and mental health problems has been recommended (DHHS, 1981; Green, 1994; Zuckerman and Parker, 1995). Children of alcohol-using mothers have been found to have higher rates of medial care visits, including visits for serious injuries, compared with nondrinking mothers (Bijur et al., 1992). A survey of 559 mothers of young children presenting for routine pediatric primary care found that $17 \%$ of women reported alcohol use problems and that close to $90 \%$ of mothers overall believed that heavy drinking impacts child health (Kahn et al., 1999). That same survey found that over $85 \%$ of women would be receptive to pediatrician screening for alcohol use problems.

The overlap of alcohol use problems and depression in women is also noteworthy in the context of child health care outcomes given that women with alcohol abuse problems display much higher rates of depression when compared with women in the general population (Grella, 1997). Both alcohol misuse and depression, especially 
when cooccurring, can negatively impact a variety of emotional, health, and self-care factors for both women and their children (Homish, 2004). Mothers with high levels of depressive symptoms engage less often in preventative practices regarding their children, such as car seat use, administering vitamins, and proper vaccination of their children (Leiferman, 2002; Turner et al., 2003).

Like other behavioral and mental health service needs, maternal alcohol use problems are not likely to be recognized by health care providers, especially given that maternal use of outpatient services drops significantly following postpartum care (Kahn et al., 1999). The emergency department (ED) generally has proven to be a productive venue for identification of alcohol use problems that may benefit from intervention. Alcohol use screening studies of adults seeking ED services for injuries have found that $20 \%$ to $30 \%$ screen positive for alcohol problems (Cherpertil, 1995). Individuals with alcohol use problems have been shown to produce greater health care costs (Holder and Blose, 1991). To our knowledge, there are no studies documenting rates of maternal alcohol use problems in the context of seeking pediatric emergency services. The pediatric ED may be a useful setting for identifying important unmet service needs for women with children for several reasons: (1) mothers of young children are not likely to use outpatient primary or specialty care services for themselves and therefore are not likely to have alcohol use problems detected, (2) maternal drinking may be associated with child health care use outcomes and indirectly to child health, and (3) a pediatric ED visit may be associated with a heightened awareness of health issues and may be an opportune time for detection and intervention.

The present study aimed to determine the prevalence of alcohol use problems as measured by a validated alcohol screening measure (TWEAK) for mothers of young children seeking pediatric emergency services, to add to the literature documenting the feasibility of using the pediatric ED to identify alcohol use problems for women, and to examine the relationship of maternal alcohol use problems with selected child health care use indicators. As alcohol abuse often cooccurs with depression and prior work has demonstrated that maternal depression is associated with child health care use outcomes (Flynn et al., 2004), depression measures were included in the analyses.

\section{METHODS}

\section{Participants and Procedures}

Participants were 361 women aged 18 years or older, who were seeking care for their children in a university hospital pediatric emergency department (PED). Eligibility was determined by a research assistant who examined the intake log that detailed the child's age, briefly stated reason for visit, and level of trauma. Women who brought a child aged 7 years or younger were approached in examination rooms at appropriate times or while seated in the waiting area. Additionally, women were not eligible for the study if they were not English speaking. If the child was brought to the ED for a severe and/or life-threatening trauma, the mother was excluded. All recruitment occurred between the hours of 12:00 PM and 12:00 AM. After written consent was obtained, each woman was self-administered the survey. If preferred by women, a research assistant read the survey to the mother, allowing women with reading difficulties to participate. The survey instrument contained measures of demographic information for both mother and child, as well as items assessing child health care utilization and maternal alcohol and depression risk.

\section{Measures}

The TWEAK is a 5-item screening tool for alcohol use problems that was used in this study (Russell, 1994). The TWEAK has been found to demonstrate good sensitivity $(0.87-0.96)$ and specificity $(0.79-0.87)$ as a screener for risk drinking in women (Bradley et al., 1998; Chan et al., 1993). Based on its brevity and validity, TWEAK is well suited as a screening instrument in a busy ED setting (Cherpertil, 1995). Both a total severity score (sum of the 5 items) and a categorical cutoff score were calculated. Our version of TWEAK used the Tolerance item that asks women to report on how many drinks they can hold; responses of more than 5 drinks received a score of 2 . Based on validation studies, positive responses to the remaining 4 items were scored and summed to derive a total TWEAK score in the following manner: $W$ ithdrawal, 2 ; Eyeopener, 1; Amnesia, 1; and need to Cut down $(K / \mathrm{C})$, 1. A summed score of 2 or more on the total TWEAK was used as the cut point in the analyses (Dawson et al., 2001; Russell, 1994). The TWEAK was completed only by women who indicated that they had any alcohol at all in the past year, and all items assessed the past 1-year time frame. Based on a screening study for risk drinking in pregnancy, Dawson and colleagues found that the cut point of 2 or more on TWEAK was associated with a sensitivity of $70 \%$ in predicting highrisk drinking and recommended this cut point as the high-risk drinking threshold. To identify moderate-risk women, Dawson et al. recommended a cut point of 1 or more on TWEAK. The frequency of women scoring above the 1 or more cut point was also calculated.

Depressive symptomatology over the past 7 days was measured using the 20-item Center for Epidemiological Studies-Depression (CES-D) scale (Corcoran and Fisher, 1987). The CES-D has been found to have adequate sensitivity $(0.80)$ for identifying a diagnosis of major depression based on an interview using the Structured Interview for the DSM-III-R in a primary care population (Fechner-Bates et al., 1994). The standard cut point of 16 was used to determine elevated symptomology. This cut point has been used as an indicator of clinically significant elevated depressive symptomatology in community samples (Myers and Weissman, 1994) as well as with pregnant women (NICHD, 1999). Child health and child health care utilization were assessed with 4 items that asked about (1) maternal ratings of overall child health (using a Likert scale ranging from "poor" to "excellent"), (2) the presence of a child's chronic illness, (3) number of visits to the ED for this particular child in the past 6 months (excluding the screening visit) and whether any of the visits resulted in admission to the hospital, and (4) whether the mother had missed a pediatric outpatient appointment in the past year. One item assessing whether or not women were currently receiving any counseling, psychotherapy, or medication for any mental health problems was included. The entire screen took approximately 10 to 15 minutes to complete. The study protocol, screening materials, and the informed consent document were approved by the University of Michigan Institutional Review Board.

\section{Data Analysis}

The primary variable of interest in this study was TWEAK, and analyses included both continuous (sum of items) and dichotomous (using the cutoff of 2) scores. Bivariate analyses using dichotomous 
variables were conducted using chi-square; $t$-tests were conducted for continuously scored variables and $p$ values at 0.05 or less were considered to be statistically significant. Bivariate logistic regression was used to examine the relationship of TWEAK and other predictors to recent pediatric ED visits (any PED visits in the past 6 months vs none, not including the screening visit), while controlling for the effect of child chronic illness. Based on previous research investigating the relationship of depression with PED use (Flynn et al., 2003) and the positive zero-order bivariate relationship of CES-D with ED use in this sample $(r=0.16, p<0.01)$, the model also included CES-D score as a predictor. The following demographic variables were considered for inclusion in the model based on their bivariate correlations with the PED use variable: maternal age, educational attainment, employment status, number of other children living in the home, and total household income. Only maternal age $(r=-0.23, p<0.001)$ and educational attainment $(r=-0.19$, $p<0.001)$ showed significant point biserial correlations with PED use and thus were included in the regression model. The demographic variables, child chronic illness, and CES-D scores were entered simultaneously into a first step of the model. TWEAK score was then entered in a second step to examine the influence of TWEAK on PED use above and beyond the effects of these other variables.

\section{RESULTS}

Of women approached, the $90 \%$ agreed to complete the screening questionnaire. Selected demographic characteristics are presented in Table 1. Most of the women were married or had a live-in partner and reported completing at least some college or higher educational attainment. The racial/ethnic background of participants reflected the demographics of the local geographic area based on the U.S. Census (2000). The mean age of mothers screened was 31 years $(\mathrm{SD}=8.3)$, and the mean age of the child brought to the PED was 2.6 years $(\mathrm{SD}=2.2)$. The median annual gross household income among the sample was $\$ 65,000$ and the mode was $\$ 100,000$ or more (range $=$ below $\$ 2,000-100,000$ or more). Based on 2005 Federal Poverty Guidelines (USDHHS, 2005), 11\% of the sample

Table 1. Demographic Characteristics of Mothers Screened in Pediatric Emergency Department $(n=361)$

\begin{tabular}{lr} 
Maternal completed years of education & \\
$\leq 8$ th grade & $0.8 \%$ \\
Ninth to 11th grade & $7.3 \%$ \\
High school graduate & $20.5 \%$ \\
Some college & $31.2 \%$ \\
College graduate & $21.1 \%$ \\
Beyond college & $19.1 \%$ \\
Mother working outside the home & $59.3 \%$ \\
Marital status & \\
Married or live-in partner & $83 \%$ \\
Not in relationship & $9.7 \%$ \\
Divorced & $4.7 \%$ \\
Separated & $0.8 \%$ \\
Widowed & $0.3 \%$ \\
Race/ethnicity & \\
Caucasian & $74 \%$ \\
African American & $14 \%$ \\
Asian American & $4.4 \%$ \\
Hispanic/Latina & $3.6 \%$ \\
Other race/ethnicity & $3.0 \%$ \\
\hline
\end{tabular}

Table 2. Rates of Response to Each TWEAK Item ${ }^{\mathrm{a}}$

\section{Can hold $>5$ drinks}

Friends or relatives have complained or worried about your drinking

Took a drink in the morning

Friend or family member told you things you said or did while you were drinking that you could not remember

Sometimes felt the need to cut down on your drinking

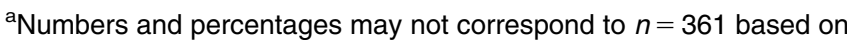
missing data on individual items and rounding of percentages.

reported annual gross household incomes at or below poverty levels. The mean number of children living in the home was $2.2(\mathrm{SD}=1.3)$. The majority of presenting complaints were related to acute or chronic illness $(58 \%)$, followed by injury/accident (19\%), pain (10\%), follow-up care $(8 \%)$, poisoning $(3 \%)$, and behavioral complaints $(2 \%)$.

Most women in the sample (65\%) reported that they had consumed alcohol in the past year. Among the total sample of $361 \%, 7 \%(n=25)$ scored above the TWEAK cutoff score of 2, indicating risk for alcohol use problems. Using a TWEAK cutoff score of $1 \%, 14 \%$ of women screened scored above that cutoff. The mean summed TWEAK score for the sample was $0.22(\mathrm{SD}=0.74)$, and summaries of responses to each of the 5 TWEAK items are presented in Table 2. Among women who scored above the cutoff on TWEAK $(\geq 2), 44 \%$ had an elevated CES-D score, compared with $25 \%$ of women below the TWEAK cutoff with elevated CES-D $\left[\chi^{2}(1)=4.1, p<0.05\right]$. The majority of mothers overall $(87.5 \%)$ rated the child's health as good to excellent, although $27 \%$ of mothers indicated that their child suffered from a chronic health condition. No significant differences between women with and without elevated TWEAK $(\geq 2)$ were found on these child health ratings. Many women indicated that they had missed an outpatient pediatric appointment for their child in the past year $(15 \%)$ and had visited the ED more than once in the past 6 months $(40 \%)$.

\section{Relationship Between TWEAK and Child Health Care Use}

Women with elevated TWEAK $(\geq 2)$ visited the PED significantly more often in the past 6 months (excluding the screening visit) than women without elevated TWEAK [mean TWEAK $\geq 2=1.3$ visits $(\mathrm{SD}=1.3)$, mean TWEAK $<2=0.68$ visits $(\mathrm{SD}=1.1), \quad t(1,268)=6.9, \quad p<0.01]$. Among women with elevated TWEAK, $8 \%$ reported that they were receiving any current mental health counseling. Despite increased PED use in women with an elevated TWEAK $(\geq 2)$, there were no significant mean differences in ratings of child's overall health found between women with and without an elevated TWEAK score. A greater percentage of women with elevated $(\geq 2)$ TWEAK $(20 \%)$ reported missing a recent pediatric outpatient appointment 
Table 3. Multivariate Logistic Regression Analyses Predicting Any Pediatric ED Use in the Past 6 Months Excluding the Screening Visit $(n=361)$

\begin{tabular}{|c|c|c|c|c|}
\hline Predictor variable & $B$ & Odds ratio & $95 \% \mathrm{Cl}$ & $p$ Value \\
\hline \multicolumn{5}{|c|}{ Model $1\left[\chi^{2}(2)=52.7, p<0.001\right]$} \\
\hline Child chronic illness & 1.50 & 4.50 & 2.64 to 7.60 & $<0.01$ \\
\hline Maternal age & -0.05 & 0.95 & 0.92 to 0.98 & $<0.01$ \\
\hline Maternal education & -0.16 & 0.85 & 0.69 to 1.06 & 0.14 \\
\hline CES-D score & 0.02 & 1.03 & 0.98 to 1.03 & 0.64 \\
\hline \multicolumn{5}{|c|}{ Model $2\left[\chi^{2}(1)=6.02, p<0.05\right]$} \\
\hline Child chronic illness & 1.51 & 4.56 & 2.67 to 7.77 & $<0.01$ \\
\hline Maternal age & -0.05 & 0.95 & 0.92 to 0.98 & $<0.01$ \\
\hline Maternal education & -0.14 & 0.87 & 0.70 to 1.08 & 0.21 \\
\hline CES-D score & 0.01 & 1.0 & 0.99 to 1.01 & 0.92 \\
\hline TWEAK score & 0.42 & 1.53 & 1.07 to 2.10 & 0.02 \\
\hline
\end{tabular}

CES-D, Center for Epidemiological Studies Depression Scale; ED, emergency department; $\mathrm{Cl}$, confidence interval.

than women without elevated TWEAK (14\%); however, this difference was not statistically significant.

Results of the bivariate logistic regression predicting PED use are presented in Table 3. In the first step of the model, child chronic illness and younger maternal age were found to significantly increase the odds of visiting the PED in the past 6 months. CES-D Scale scores and maternal education were not significant predictors. With TWEAK included as a predictor in the second step of the model, child illness, higher TWEAK score, and younger maternal age significantly predicted PED use in the past 6 months.

\section{DISCUSSION}

This study examined the feasibility of screening for maternal alcohol use problems in a PED setting as well as the prevalence of alcohol use problems. Ninety percent of mothers approached in a busy pediatric ED agreed to complete the TWEAK. This screening tool is ideally suited for ED settings given both its brevity and its sensitivity in detecting high-risk alcohol use in childbearing-aged women. Consistent with previous research (Larkby and Day, 1999), approximately 1 in 10 women (depending on the TWEAK cutoff score used) screened were found to be at risk for alcohol use problems. These results indicate that risk for maternal alcohol use problems occurs at a significant rate within PED settings and that screening in this manner is likely to result in feasible and accurate detection. Women with elevated TWEAK $(\geq 2)$ more frequently utilized the PED for child health care, despite the fact that they did not report any significant differences in overall health status in their children when compared with women who were not at risk for alcohol use problems. The relationship of TWEAK with child health care use outcomes provides preliminary support for its construct validity as a screener for maternal drinking in relation to child health care use outcomes.
As expected, we also found a significant relationship between the presence of a child's chronic illness and increased PED use. However, higher TWEAK scores significantly increased the odds of visiting the PED even when child health status was included in the multivariate model. Consistent with previous reports, younger maternal age was also related to a greater likelihood of PED use (Watson and Kemper, 1995). Among women with elevated TWEAK $(\geq 2)$, nearly half also showed elevated depressive symptoms, significantly more so than women without elevated TWEAK. Taken together, these results suggest that alcohol use risk is an important factor to include among recommended screening for an array of maternal behavioral and mental health needs in pediatric settings (Green, 1994) and that TWEAK may be a suitable screening measure that is associated with other mental health and child outcomes. The rate of compliance with alcohol screening as part of usual care in this kind of setting cannot be determined from this study because screening was conducted as part of the research study. However, routine pediatric setting screening for parental alcohol use and related behavioral and psychosocial risk factors has been recommended by the American Academy of Pediatrics (1993). One study found that over $90 \%$ of heavy alcoholusing mothers welcomed or would not mind inquiries about alcohol use in the pediatric setting.

Screening for these issues is an important initial step in identifying women who may benefit from further follow-up and assessment and whose children may be experiencing consequences directly or indirectly related to alcohol use. The precise mechanism of the link between maternal drinking and child health care use cannot be determined from this study. For example, it is premature to speculate about increased risk of injury or illness directly tied to maternal drinking. However, maternal drinking has previously been linked to increased child injury requiring medical care (Bijur et al., 1992). Although 14\% of women scored above the more conservative TWEAK cutoff score ( $>1$, indicative of moderate alcohol use risk), it is possible that TWEAK may have missed a percentage of women using alcohol at levels that may also have consequences for mother and child. Future studies should include more detailed assessments of alcohol use behaviors and diagnosis, in addition to comprehensive assessments of child health care outcomes that may be related to alcohol use. The inclusion of child medical record data in future studies is recommended. Irrespective of the mechanism of this relationship, this study demonstrated that screening for alcohol use problems is feasible in pediatric ED and that screening can identify approximately $10 \%$ of mothers who may be at risk and in need of intervention. As most $(92 \%)$ were not receiving any mental health counseling, this may represent an unmet service need for this population.

Another limitation of this study was the exclusion of mothers of children being seen for the most severe illness and injuries and mothers who did not present for care at 
the PED at all. Therefore, rates of alcohol use problems and links to child health care among those women are not known. Given the emergent nature of more severe presenting complaints and presumed accompanying distress, it is not known whether screening for alcohol use problems would be feasible in those situations. These results may also not generalize to ED settings comprised mainly of racial and ethnic minority women or uninsured women who may face numerous psychosocial difficulties (such as poverty and lack of health insurance) impacting child health care issues. This sample included a full range of income levels, with a minority reporting household incomes below the poverty level. This implies that screening should be conducted with women across the income spectrum. Although we did not find a relationship between household income and PED use, results may be different among samples comprised mainly of lower income women.

This study represents a preliminary investigation of a possible link between maternal alcohol problems and child health care use and demonstrates the potential value of pediatric ED settings for screening and research. Previous research with other populations has demonstrated that screening for alcohol risk in adult ED settings may be successfully combined with intervention strategies to improve an array of alcohol use outcomes (Longabaugh et al., 2001). A further understanding of child use outcomes associated with identified maternal drinking is an important preliminary step toward effective intervention.

\section{REFERENCES}

American Academy of Pediatrics, Committee on Substance Abuse and Committee on Children with Disabilities (1993) Fetal alcohol syndrome and fetal alcohol effects. Pediatrics 91:1004-1006.

Bijur PE, Kurzon M, Overpeck MD, Scheidt PC (1992) Parental alcohol use, problem drinking, and children's injuries. JAMA 267:3166-3171.

Bradley KA, Boyd-Wickizer J, Powell S, Burman ML (1998) Alcohol screening questionnaires in women: a critical review. JAMA 280: 166-171.

Chan AW, Pristach EA, Welte JW, Russell M (1993) Use of the TWEAK test in screening for alcoholism/heavy drinking in three populations. Alcohol Clin Exp Res 17:1188-1192.

Cherpertil CJ (1995) Screening for alcohol problems in the emergency department. Ann Emerg Med 26:158-166.

Conners NA, Bradley RH, Mansell LW, Liu JY, Roberts TJ, Burgdorf K, Herrell JM (2004) Children of mothers with serious substance abuse problems: an accumulation of risks. Am J Drug Alcohol Abuse 30:85-100.

Corcoran K, Fisher J (1987) Measures for Clinical Practice: A Sourcebook. Free Press, New York.

Dawson DA, Das A, Faden VB, Bhashar B, Krulewitch CJ, Wesley B (2001) Screening for high- and moderate-risk drinking during pregnancy: a comparison of several TWEAK-based screeners. Alcohol Clin Exp Res 25:1342-1349.
Fechner-Bates S, Coyne JC, Schwenk TL (1994) The relationship of self reported distress to depressive disorders and other psychopathology. J Consult Clin Psychol 62:550-559.

Flynn HA, Davis M, Marcus SM, Cunningham R, Blow FC (2004) Rates of maternal depression in pediatric emergency department and relationship to child service utilization. Gen Hosp Psychiatry 26: 316-322.

Green M (1994) Bright Futures: National Guidelines for Health Supervision of Infants, Children and Adolescents. National Center for Education in Maternal and Child Health, Arlington, VA.

Grella CE (1997) Services for perinatal women with substance abuse and mental health disorders: the unmet need. J Psychoactive Drugs 29:67-78.

Harter SL (2000) Psychosocial adjustment of adult children of alcoholics: a review of the recent empirical literature. Clin Psychol Rev 20: 311-337.

Holder HD, Blose JO (1991) A comparison of occupational and nonoccupational disability payments and work absences for alcoholics and nonalcoholics. J Occup Med 33:453-457.

Homish GG, Cornelius JR, Richardson GA, Day NL (2004) Antenatal risk factors associated with postpartum comorbid alcohol use and depressive symptomatology. Alcohol Clin Exp Res 28:1242-1248.

Kahn RS, Wise PH, Finkelstein JA, Bernstein HH, Lowe JA, Homer CJ (1999) The scope of unmet maternal health needs in pediatric settings. Pediatrics 103:576-581.

Larkby C, Day N (1999) The effects of prenatal alcohol exposure. Alcohol Health Res World 21:192-198.

Leiferman J (2002) The effect of maternal depressive symptomatology on maternal behaviors associated with child health. Health Educ Behav 29:596-607.

Longabaugh R, Wollard RE, Nirenberg TD (2001) Evaluating the effects of a brief motivational intervention for injured drinkers in the emergency department. J Stud Alcohol 62:806-816.

Myers JK, Weissman MM (1980) Use of a self-report symptom scale to detect depression in a community sample. Am J Psychiatry 137: 1081-1084.

National Institute of Child Health and Human Development (NICHD). (1999) Chronicity of maternal depressive symptoms, maternal sensitivity, and child functioning at 36 months. Dev Psychol 35: 1297-1310.

Richter L, Richter D (2001) Exposure to parental alcohol use: effects on children's health and development. Am J Orthopsychiatry 71:182-203.

Russell M (1994) New assessment tools for risk drinking during pregnancy: T-ACE, TWEAK and others. Alcohol Health Res World 18:55-61.

Sher KJ (1991) Children of Alcoholics: A Critical Appraisal of Theory and Research. University of Chicago Press, Chicago.

Turner C, Boyle F, O'Rourke P (2003) Mothers' health post-partum and their patterns of seeking vaccination for their infants. Int $\mathbf{J}$ Nurs Pract 9:120-126.

U.S. Department of Heath and Human Services. (1981) Select Panel for the Promotion of Child Health, Better Health for Our Children: A National Strategy. USDHHS Publication No. 79-550-71.

U.S. Department of Heath and Human Services. (2005) The 2005 HHS poverty guidelines. Fed Regist 70:8373-8375.

Watson JM, Kemper KJ (1995) Maternal factors and child's health care use. Soc Sci Med 40:623-628.

Zuckerman B, Parker S (1995) Preventive pediatrics - new models of providing needed health services. Pediatrics 95:758-762. 